\title{
Toutes ensemble? Femmes et société: intégration et marginalisation
}

\section{Introduction}

Les trois articles inclus dans cette section spéciale s'articulent autour de la question de la place des femmes dans la société, dans la lignée du thème initial du colloque Women in French de 2013, dont ils sont issus: "Solitaires, solidaires ». Plus précisément, pour ce qui est des études présentées ici, se pose la question des structures qui favorisent, ou non, l'intégration des femmes dans la société contemporaine: qu'il s'agisse de la famille, des réseaux professionnels et associatifs, des activités professionnelles et créatives, ou de l'ancrage de l'autonomie au féminin dans les mentalités et les habitudes.

La place des femmes dans la société est un sujet en constante évolution qui a pris une place considérable dans les débats publics. En France, la loi égalité homme-femme, portée par Najat Vallaud-Belkacem, alors ministre en charge des droits des femmes, a été adoptée le 4 août $2014^{1}$ et s'étend à plusieurs sphères d'action: la valorisation des pères, en leur permettant de prendre un congé parental; l'égalité hommes-femmes dans les milieux professionnels, au niveau des salaires en particulier ainsi que pour promouvoir la présence de femmes dans les sphères «à haute responsabilité » de la société; la protection des mères isolées afin qu'elles ne se trouvent pas en situation financière précaire; la lutte contre le sexisme et les violences contre les femmes. Il est trop tôt pour savoir si ce dispositif législatif va permettre, à long terme, de rééquilibrer une situation dans laquelle de nombreuses femmes renoncent à une activité professionnelle lorsqu'elles deviennent mères, tandis que celles qui travaillent continuent à percevoir, en moyenne, des salaires inférieurs à ceux des hommes, du fait qu'elles ont tendance à occuper les postes professionnels les plus précaires et qu'à poste égal de nombreuses discriminations ont encore lieu. Par ailleurs, tant que le statut de mère au foyer continuera à être dénigré et dévalorisé, tant que les médias continueront à véhiculer des stéréotypes qui touchent tout autant la «masculinité » que la «féminité », tant que les filles ne seront pas encouragées par tous - famille, proches, professeurs - à se diriger vers des filières dites «masculines", tant que les violences contre les femmes resteront sans conséquences réelles, de telles initiatives seront insuffisantes. Certes, une loi seule ne suffit pas à faire changer les mentalités. Mais elle peut intervenir à un moment où, dans un certain pays ou à travers les frontières, se dessinent des envies de changement, comme on le voit depuis le début des années 2000 avec la légalisation du mariage homosexuel, adoptée par de nombreux pays dont le nombre ne cesse de grandir, avec des disparités criantes à travers le monde. Ces batailles se jouent sur plusieurs fronts: la mise en place de lois touchant à des domaines perçus comme personnels, voire intimes - le rapport aux enfants et à la famille, les relations de couple et les séparations, la puissance morale du harcèlement, la sexualité montre bien que, plus que jamais, notre société opère sur une abolition des cloisonnements entre sphère publique et institutionnelle d'une part, et vie privée de l'autre.

Cette section spéciale, qui comporte trois articles, n'a aucune prétention à couvrir un sujet non seulement vaste tant il touche à des domaines variés, mais aussi en mutation constante. Nous avons choisi de grouper une série d'études de cas sur des femmes ayant fait l'expérience de la marginalisation et l'isolement (volontairement ou non) au sein d'un groupe

\footnotetext{
${ }^{1}$ Le texte de loi est disponible sur $<$ http://www.legifrance.gouv.fr/affichTexte.do?cidTexte=JORFTEXT0000 29330832\&categorieLien $=\mathrm{id}>$.
}

Pour une vue d'ensemble de cette loi, voir $<$ http://www.gouvernement.fr/ action/la-loi-pour-l-egalitereelle-entre-les-femmes-et-les-hommes $>$ [consultés le 25 août 2015]. 
et/ou de la société dans laquelle elles vivent, qu'elles soient célibataires dans une société toujours dominée par les valeurs patriarcales (Charpentier, dans son article sur les femmes seules au Maghreb), mères célibataires en France à la fin du vingtième siècle (Garcia) ou femmes «entrepreneures » qui tentent de combiner les contraintes de leur «business » à celles de leur vie de famille (Landour). Face à ces situations, quelles structures légales, associatives ou autres sont en place pour les soutenir? Quels discours et croyances entourent ces structures et ont-ils un impact sur les représentations et stéréotypes de genre? Quels réseaux peuvent être mis en place pour aider les femmes mères/célibataires à sortir de l'isolement et, de manière plus importante encore, quels outils et mécanismes sont à la disposition de ces femmes pour leur éviter de se retrouver encore plus marginalisées? Les structures de soutien et de solidarité, lorsqu'elles existent, sont-elles même souhaitables ou bénéfiques?

Pour les «Mompreneurs » interviewées par Landour, l'idée que l'activité professionnelle mène à plus d'autonomie et de satisfaction est remise en question - ces activités pouvant en effet non seulement mener à une marginalisation accrue mais aussi renforcer les stéréotypes de genre. Dans le cas des femmes célibataires du Maghreb interviewées par Charpentier, bien que la littérature et la créativité leur offrent une possible émancipation, leur choix - celui de valoriser leur trajectoire professionnelle aux dépens d'une vie de famille - demeure inédit et difficile à assumer au grand jour. Enfin, même si l'étude de Garcia montre que les mères célibataires ne sont plus marginalisées comme elles l'étaient auparavant en France, elles sont toujours confrontées à un certain nombre de défis et d'idées reçues, y compris les leurs, dans leur quête d'émancipation et d'autonomie.

Les approches sociologiques des contributrices à cette section spéciale, toutes basées sur des enquêtes de terrain et des entretiens menés par les chercheuses, donnent à lire des récits qui, en donnant une voix à des catégories socio-professionnelles souvent tues, vont au-delà du stéréotype. Par ailleurs, ces articles révèlent les dynamiques complexes qui sont en jeu dans la question de l'émancipation féminine, en particulier, les tensions entre sphères privée et publique. Les articles de Garcia et Charpentier montrent ainsi à quel point la situation des mères seules en France et celle des femmes célibataires au Maghreb n'est pas fondée sur une division privée/publique clairement définie, mais sur des processus de marginalisation et d'intégration qui dépendent de tensions complexes entre les aspirations personnelles, les circonstances matérielles, les pressions familiales et l'existence - ou l'absence - de réseaux de soutien. L'article de Landour permet également de nuancer ces mouvements et tensions qui s'expriment de manière aigüe pour les femmes qui essaient de maintenir une activité professionnelle depuis leur environnement domestique.

En s'intéressant aux imbrications entre divers domaines (familiaux, professionnels, politiques, associatifs), à différents niveaux (personnels, locaux, nationaux), à des réseaux récemment créés, mais aussi au manque de structures de soutien, cette section spéciale offre un aperçu des tensions et de l'isolement rencontrés par certains groupes de femmes lors de leur parcours. Loin d'être un simple état des lieux cependant, ces articles mettent en lumière les enjeux, les combats à mener, mais aussi les outils à développer pour atteindre autonomie et solidarité entre toutes et tous.

Elise Hugueny-Léger (University of St Andrews) et Caroline Verdier (University of Strathclyde) 